\title{
IMPLICACIONES Y RETOS PARA LA FORMACIÓN EN COMUNICACIÓN PARA EL CAMBIO SOCIAL. NUEVAS LÍNEAS DE ABORDAJE.
}

IMPLICATIONS AND CHALLENGES FOR COMMUNICATION TRAINING

FOR SOCIAL CHANGE. NEW APPROACHES.

Por: Daivy Dalila Díaz Santana ${ }^{1}$

Giovanni Bohórquez Pereira²

Olga Beatriz Rueda Barrios ${ }^{3}$

Recibido: 10 de mayo de 2019 - Aprobado: 30 de septiembre 2019

\section{RESUMEN}

Con los emergentes cambios sociopolíticos en el mundo, la academia sigue siendo uno de los escenarios desde donde se deben promover acciones verdaderas y profundas que conlleven al cambio social. A partir de la revisión documental de trabajos de investigación en el área se identifican las tendencias, retos e implicaciones que la comunicación enfrenta en la promoción de experiencias y proyectos que desde la academia se proyecten a las comunidades. El presente trabajo se centra en los campos de la cultura digital con sus nuevas narrativas, la innovación social, la gobernanza y el deporte, desde los cuales se vienen abordando alternativas para generar cambios en la sociedad y se trazan caminos para, desde la formación de comunicadores sociales, construir nuevos escenarios que hagan posible vivenciar la comunicación para el cambio social e incidir en la realidad contextual, más allá de un papel instrumental, es decir, generar competencias para el análisis, la planeación y la acción de la comunicación en relación con los procesos de desarrollo social y territorial.

Palabras clave: Comunicación, cambio social, innovación social, comunicación pública, gobernanza, TIC.

ABSTRACT

With the emerging sociopolitical changes in the world, the academy still is a significant

\footnotetext{
${ }^{1}$ Magister en Educación. Docente - Coordinadora Especialización Gerencia de la Comunicación Organizacional, de la Facultad de Comunicación Social - Periodismo de la Universidad Pontificia Bolivariana Seccional Bucaramanga. https://orcid.org/0000-00032499-3425 daivy.diaz@upb.edu.co

${ }^{2}$ Magister en Ciencias Políticas de la Pontificia Universidad Javeriana, docente asociado de la Escuela en Ciencias Social, de la Facultad de Comunicación Social-Periodismo, de la Universidad Pontificia Bolivariana. https://orcid.org/0000-0001-7752-2008 giovanni.bohorquez@upb.edu.co

${ }^{3}$ Comunicadora social de la Universidad Autonoma de Bucaramanga, Magister en Comunicación, Educación y Cultura de la Universidad Autonoma de Barcelona, magister en Comunicación Digital de la Universidad Pontificia Bolivariana y Doctora en Contenidos de Comunicación Digital de la Universidad Autonoma de Barcelona. Directora de Facultad de Comunicación Social - Periodismo de la Universidad Pontificia Bolivariana, Bucaramanga. https://orcid.org/0000-0001-8539-9038 olgab.rueda@upb.edu.co
} 
scenario from which meaningful and profound actions are to be promoted, leading to social change. Through a documentary review within the field, this research identifies trends, challenges and implications that the communication confronts in order to promote experiences and projects presented by the Academia in favor of the communities. This paper focuses on the field of digital culture with its new narratives, social innovation, governance and sports. These fields serve to generate new paths that make new communication scenarios possible, thus enabling social change in real life contexts, and generating competences aiming at the analysis, planning and implementation of communication in regards to social and territorial development.

Keywords: Communication, social change, social innovation, public communication, governance, ICT.

\section{INTRODUCCIÓN}

La Comunicación para el Desarrollo y el Cambio Social (CCS) es un ámbito de actuación e investigación que está en constante apertura de sus límites, lo que le lleva a enfrentar nuevos retos y oportunidades de cara a los requerimientos, tendencias, procesos y tecnologías emergentes, que dinamizan la sociedad actual. A la luz de esto, es pertinente indagar sobre lo que es y debería ser su papel, de cara a dichos cambios y los retos sociales que implican. En consecuencia, se considera pertinente realizar un abordaje del tema a partir de los siguientes cuestionamientos.

¿Cuáles son los futuros caminos o líneas de trabajo que tienden a marcar o ceñir la comunicación en los próximos diez años y en particular la relacionada con el cambio y transformación social?

¿El campo o área de la comunicación vinculada al cambio y la transformación social debe ser el que lidere esos procesos o le corresponde mantenerse simplemente como un actor vinculante o acompañante?

Ante los interrogantes se hace necesario realizar algunas reflexiones previas de lo que ha sido el devenir de la Comunicación para el Cambio Social y posteriormente inducir caminos posibles. La primera parte se centra en reconocer qué este campo es considerado como uno de los aportes a la esencia propia de la comunicación, tanto en lo conceptual, como en sus elementos metodológicos, especialmente, en el contexto latinoamericano en el que persiste un llamado a la transformación social desde las necesidades que demandan las comunidades. La segunda está orientada a presentar cuatro interesantes campos a los cuales la Comunicación para el Cambio Social y el Desarrollo se integra, creando aleaciones estratégicas desde las que se lideren verdaderos procesos de empoderamiento de las personas sobre su realidad.

\section{Reflexiones previas}

A finales de los 50, diversos investigadores estadounidenses empezaron a trabajar en estudios 
acerca de los nuevos enfoques de la comunicación, principalmente relacionados con el desarrollo.

Daniel Lerner aseguró que la comunicación masiva era a la vez inductora e indicadora de cambio social. En los primeros pasos hacia el desarrollo, los medios informaban a la población sobre las propuestas o proyectos, mostraban las ventajas de estas iniciativas, recomendaban que sean apoyadas y esperaban la respuesta del receptor, que se evidenciaba en estadísticas. Sin duda alguna, con los medios masivos se podía llegar a grandes grupos poblacionales, de una manera general y rápida (Didis, 2011, p. 7).

No obstante, esta perspectiva otorgaba a los medios de comunicación un poder de persuasión y la posibilidad de generar estereotipos sobre los estilos de vida que poco o nada tenían que ver con las expectativas de la población, aspecto que contradice la esencia de la CCS.

Finalizada la década de los 50, la denominada Comunicación para el Desarrollo, se consolidó en las comunidades, luego del programa "Alianza para el Progreso"4. Con esa experiencia, catedráticos como Luis Ramiro Beltrán, Mario Kaplún y Antonio Pasquali, por citar solo tres del largo listado de pensadores, hicieron posible reconocer, en ese momento de la historia, que América Latina, dadas sus características sociales, económicas, políticas y culturales, requería de construcciones propias para superar sus dificultades, que necesita organización social y no individualización, que interaccionar en común-unión sus modos de pensar y proyectar salidas a sus dificultades ante gobiernos fallidos e incapaces de proporcionar condiciones y espacios de participación para la construcción de reales Estados-Nación, esto es indispensable si la búsqueda es un cambio de modelo político, social y la configuración de un nuevo modelo de comunicación.

Hacia 1962 Everett Rogers afirmó que era necesario conocer las innovaciones y adaptarse a ellas, de esta manera, la difusión consistía en un proceso de comunicación, limitándola al hecho de socializar nuevas ideas e invenciones entre los miembros de un grupo social, en un tiempo determinado, a través de diferentes canales de comunicación. Destacó la importancia de la comunicación interpersonal y el papel de las redes sociales.

Por su parte, Wilbur Schramm, estudió el fenómeno de la comunicación y su relación con el desarrollo. En 1964 publicó un estudio sobre las características de la comunicación masiva para atender las necesidades sociales de los llamados países en vía de desarrollo y determinó que la parte receptora debía "estar informada de los planes, acciones, logros y limitaciones del esfuerzo pro desarrollo; hacerse partícipe del proceso de toma de decisiones sobre asuntos de interés colectivo; y aprender las destrezas que el desarrollo les demanda dominar" (Beltrán, 2006, p. 19).

\footnotetext{
${ }^{4} \mathrm{La}$ contención del comunismo en Latinoamérica tuvo uno de sus hitos en el programa que el presidente de Estados Unidos John F. Kennedy expuso el 13 de marzo de 1961 ante el cuerpo diplomático latinoamericano, denominado «Alianza para el Progreso». Se trataba de combatir la creciente pobreza del área latinoamericana mediante la asignación de un fondo de 20.000 millones de dólares por diez años, que sería destinado a la construcción de escuelas, hospitales y otras infraestructuras. El objetivo último era promover la democracia representativa y prevenir la tentación revolucionaria. Fuente: dipublico.org. Disponible en https:/www.dipublico.org/glossary/alianza-para-el-progreso/
} 
En la década de los 70 se plantearon las primeras ideas de cómo la sociedad debería participar e involucrarse de lleno en su transformación, también fue el momento en que algunos teóricos evidenciaron y aportaron a los primeros y efectivos modelos comunicativos, que demostraban que era posible el acceso, no solo a la información, sino a la construcción y difusión desde su contexto de contenidos propios. Desde esta perspectiva, no se otorga prioridad a la estética, la técnica, o a seguir modelos, lo fundamental se centra en el mensaje, la intención y la orientación política.

Para la década de los 80 se desarrolló en América Latina el concepto de la comunicación alternativa, que pretendió promover la participación activa de las comunidades, a través de medios de comunicación no comerciales. Investigadores como Jesús Martín Barbero empezaron a trabajar en el análisis de la cultura y las mediaciones y las formas en que las audiencias apropian el contenido que circula por los medios. En este contexto, el argentino Néstor García Canclini, realizó sus aportes desde el análisis del consumismo, la globalidad y la interculturalidad en América Latina. Por su parte, Kaplún, seducido por el enfoque trazado por Paulo Freire, en una clara perspectiva de la comunicación educativa, propuso una comunicación transformadora en oposición a la comunicación bancaria. Finalmente, fueron contundentes las contribuciones de Luis Ramiro Beltrán, considerado el pionero de la Escuela Latinoamericana de la Comunicación, reconocido por sus ideas de democratización y la puesta en marcha de las Políticas Nacionales de Comunicación.

"La comunicación alternativa para el desarrollo democrático es la expansión y el equilibrio en el acceso de la gente al proceso de comunicación y en su participación en el mismo empleando los medios -masivos, interpersonales y mixtos- para asegurar, además del avance tecnológico y del bienestar material, la justicia social, la libertad para todos y el gobierno de la mayoría" (Beltrán, 2005, p. 21).

Otro elemento significativo para la consolidación de la Escuela Latinoamericana de la Comunicación fue la constitución de diversas agremiaciones de profesionales de la comunicación comprometidos con las ideas desarrollistas que aportaban desde la perspectiva de estos territorios.

(...) Asociación Latinoamericana de Escuelas Radiofónicas (ALER), que vino a sumarse a las ya existentes organizaciones católicas de prensa y medios audiovisuales. Nacieron también la Federación Latinoamericana de Periodistas (FELAP), la Asociación Latinoamericana de Investigadores de la Comunicación (ALAIC) antecedida por el precursor Instituto de Investigación de la Comunicación (ININCO) en Venezuela. Y algo después, sumándose al CIESPAL ya existente en Ecuador, surgiría también la Federación Latinoamericana de Facultades de Comunicación (FELAFACS), las que ahora pasan del millar. Además se crearon primero en México, el Instituto Latinoamericano de Estudios Transnacionales (ILET) y más tarde en Perú el Instituto para América Latina (IPAL) y el CENECA en Chile, entre otras entidades. Todas esas agrupaciones se comprometieron a fondo con el ideal de la democratización de la comunicación y del desarrollo (Beltrán, 2005, p. 22). 
Alfonso Gumucio-Dagron en Haciendo olas entregó una recopilación de 50 experiencias significativas en el mundo, algunas de ellas del orden instrumental y otras con características o elementos de transformación y cambio social y político. Las relacionadas con América Latina, mostraban de manera transparente la forma en que la sociedad, con grandes limitaciones, producía reflexiones del porqué de un cambio social. "Los ejemplos seleccionados muestran que lo extraordinario en la comunicación participativa es que puede adoptar diferentes formas, de acuerdo con las necesidades, y que no es posible imponer un modelo único sobre la riqueza de perspectivas y de interacciones culturales" (Gumucio-Dagron, 2001, pág.6).

No obstante, frente al limitado efecto de los procesos de cambio se hizo necesario revisar el liderazgo de estas iniciativas; por tanto, se consideró que gran parte de la responsabilidad del proceso social recaía sobre la(s) entidad(es) que lo implementaban o proponían. Así, proyectos agrícolas con campesinos, radios comunitarias en países latinoamericanos, telecentros, iniciativas locales de canales comunitarios o colectivos juveniles, asumían un papel paternalista, dejando de lado el empoderamiento y la participación activa de las comunidades, lo que finalmente llevaba al fracaso, dada la dependencia de organismos externos.

Ahora bien, no es la pretensión de este documento hacer el mapa cronológico de la Comunicación para el Desarrollo y Cambio Social y todas sus manifestaciones o postulados, ese ejercicio, con gran altura lo han realizado ya Beltrán (2006), Alfaro (2006), Cárdenas (2009), Navarro (2010), Herrera (2011), Angulo (2012), Cadavid y Gumucio-Dagron (2014), se pretende entonces esbozar algunas líneas de abordaje en las dimensiones actuales de la CCS.

\section{Lo que se viene haciendo}

De lo revisado hasta el momento, en lo relacionado a procesos sociales liderados por las Facultades de Comunicación Social-Periodismo del país, se encuentran altibajos, logros, frustraciones y experiencias, que hacen parte del aprendizaje alcanzado. Los resultados dejan ver que el impacto social no se logra fácilmente, pues la tendencia ha sido más del orden formativo que de liderazgo para la acción social. Sin embargo, no por ello se pueden obviar o dejar de lado trabajos de referencia como los liderados por Pereira (2005), Roveda (2006), Álvarez (2009), Herrera y Uruburu (2010), Fuentes (2010), Rueda (2006), Bohórquez-Pereira (2013), entre otros, quienes en su momento expusieron en el orden nacional y regional, el panorama y la situación del campo de estudio, alcances y perspectivas.

En este orden de ideas, se registran a continuación estudios de facultades colombianas que vienen realizando trabajos orientados a la generación de conocimiento científico, a partir de procesos de intervención y cambio social. 
En primer lugar, Mendivil, Racedo, Meléndez y Rosero (2015) abordan los usos de la comunicación en su papel de dinamizador de la cultura en procesos de empoderamiento y participación en contextos de violencia, a partir de las posibilidades de hacer público el dolor, decantar procesos de duelo y generar alternativas de encuentros comunitarios para cohesionar sus visiones sobre el futuro.

En este mismo sentido los docentes Canal, Navarro y Camargo (2016), presentan a la comunicación desde una perspectiva de género que aboga por una democracia legítima, por el respeto, la tolerancia, la igualdad y la inclusión social desde las narrativas emergentes en comunidades palafíticas del Caribe colombiano que han sido víctimas del desplazamiento forzado.

Se suman dos estudios realizados en la comunidad de Palenque, en el departamento de Bolívar. En primer lugar, Navarro-Díaz (2017), expone experiencias inéditas de las formas de organización juvenil y sus prácticas de comunicación, desde una relación dialógica con los participantes y entre ellos. El autor se enfoca en esas "otras" formas de comunicación que son usadas por los jóvenes para interlocutar entre ellos y con su contexto.

Por su parte Navarro-Díaz y Aguilar (2015), develan cómo acciones comunicativas concretas han generado dinámicas de transformación, fortalecimiento, recuperación, preservación y resistencia en el tejido social de la comunidad vulnerada desde lo socioeconómico y que representa un patrimonio cultural para la sociedad colombiana. En el estudio se sistematiza la forma como los jóvenes de esta comunidad encuentran en la oralidad y la memoria histórica, con sus diferentes manifestaciones artísticas asociadas a la danza y al canto, formas de comunicación, las cuales ocurren en gran medida en las calles, siendo estos escenarios propicios para la reafirmación de su cultura y la expresión de la forma como ven y entienden el contexto que les envuelve.

Mejía-Betancur (2014) en una investigación sobre el arte como herramienta de transformación sociocultural en las comunas de Medellín, Antioquia, una comunidad altamente golpeada por la violencia y la marginación, a través de un trabajo llevado a cabo con grupos artísticos, del que participan personas que tuvieron que vivir de cerca el tema de la ilegalidad como producto del narcotráfico en el país, permite ver como la comunicación para el cambio social se integra con el arte en un proceso de la transformación sociocultural para la recuperación de valores.

Dentro del contexto de violencia que ha demarcado en gran medida la dinámica socioeconómica del país, Forero, Hernández y Ordoñez (2015), presentan un estudio realizado con Parceleros de Santa Paula, beneficiarios de la política de restitución de tierra en el departamento de Córdoba, en el que se describe el papel que juegan la comunicación y el tejido social en la comprensión de la construcción de la paz en el ámbito local. La comunicación les permite constituirse en líderes del 
proceso de reparación al interior de sus núcleos familiares y sus comunidades.

\section{¿Hacia dónde vamos?}

Tomás Villasante (2015) en su texto Metodologías ¿para qué? ¿Para quién?, recomienda hacerse estos cuestionamientos antes de proceder a tomar decisiones desde el mundo de la investigación, no resulta desproporcionado utilizarlas en este momento para la reflexión del camino a seguir en materia de comunicación para el cambio social.

Quien se hace las preguntas epistemológicas básicas, ¿para quién?, ¿para qué es todo este proceso?, puede ser consciente de dónde se mete, y puede prever las formas de contrarrestar los efectos potencialmente perjudiciales. Para alcanzar un grado mayor de "objetivización" colocará controles y métodos que le acerquen a una realidad lo más operativa posible. Por eso valoramos mucho las metodologías participativas, porque parten de tener en cuenta a los diferentes actores sociales que tienen intereses en los procesos de investigación o de intervención. Porque antes de plantearse técnicas de tipo cualitativo o cuantitativo, se planean los problemas previos: ¿Quién manda aquí? ¿Cómo se pueden contrarrestar los efectos negativos de los intereses en juego? No es solo una cuestión de ética o de ideología, es una cuestión metodológica básica la que planteamos (Villasante, 2017, pág. 215 -216).

Pues bien, el primer llamado entonces es preguntar a quiénes se van a orientar las propuestas de nuevos campos desde los que se aborda la comunicación para el cambio social en las facultades ¿Se pretende tomar a las comunidades desde una perspectiva instrumental o, por el contrario, generar competencias para el abordaje del campo desde las realidades de las comunidades aportando a su desarrollo y transformación social?

En este sentido es pertinente citar a Barranquero (2005) quien afirma que "(...) el "paradigma participativo" de la comunicación para el cambio social se caracteriza por su capacidad de involucrar a la sociedad civil en su propio proceso de transformación a través de la comunicación, la cultura y la educación" (pág.243). De igual forma, Kaplún (1985) expone que "una cosa es la seudocomunicación que busca dominar e imponer, conservar el control y el monopolio del habla para mantener a la sociedad pasiva y sometida a estructuras injustas; y otra bien distinta la comunicación que se propone generar un diálogo democrático, participativo e igualitario que contribuya a cambiar esa sociedad y a dinamizar el compromiso social” (pág. 112).

Un segundo aspecto para tener presente es superar la mirada de la Comunicación para el Desarro1lo, como instrumento de apoyo a un modelo económico. Al respecto Cadavid (2014) comprende a la Comunicación como campo de construcción social y cultural para transformar esa sociedad en su conjunto. Esto implica integración, participación, deseos; por ende, cultura y el aprovechamiento de los recursos y tecnologías con los que cuentan. En palabras de la autora este concepto 
es más acción política que conceptual. Por consiguiente “(...) el campo de la comunicación para el cambio social asume y da perfil a nuevos sujetos sociales surgidos de procesos de movilización y lucha por el reconocimiento" (Cadavid, 2014, pág. 42).

Es tan real lo planteado hasta el momento que al interior de los Objetivos del Desarrollo del Milenio (2000-2015) estuvo presente el papel activo de la comunicación. Durante quince años esta hizo parte de una política mundial. Así quedó planteado en el Octavo Objetivo: "Fomentar una alianza mundial para el Desarrollo" y en particular, trabajar por la cooperación “...con el sector privado, hacer más accesible los beneficios de las nuevas tecnologías, especialmente las de información y comunicaciones", aspectos en los que la comunicación hoy sigue trabajando. (FAO, 2013). Desde el año 2015 se proclamaron los Objetivos del Desarrollo Sostenible, ODS, un plan de acción en favor de las personas, el planeta y la prosperidad, son 17 posibilidades que requieren ser estudiadas desde la comunicación para el cambio social y de esta manera concreta involucrase, esta vez menos desde el modelo de difusión y más hacia un modelo de participación.

\section{Campos de investigación}

Recientemente Montero-Díaz, Cobo, Gutiérrez-Salcedo, Segado-Boj \& Herrera-Viedma (2018), han expuesto en su artículo Mapeo científico de la categoría comunicación en WoS $S^{5}$ que temas prospectivos de interés para los científicos sociales estarían en: conflicto, juventud y activismo en internet. Lo que coincide con los resultados de indagación sobre los nuevos campos de actuación de la CCS en que Internet como comunidad y espacio virtual de relaciones ha ido ganando relevancia como objeto de estudio, en la medida que la democracia y la opinión pública, también tienen lugar en dichos espacios virtuales lo que ha llevado a que en los últimos años haya aumentado el interés en estudiar las nuevas narrativas y espacios de interacción que se configuran en la redes digitales (Sforzin, 2016; Flores-Marques, 2016).

Se tiene claridad sobre la necesidad de seguir indagando sobre los nuevos planteamientos y/o modelos sociales que buscan transformar y generar oportunidades para El buen vivir (Barranquero y Sáez, 2015) de todos los que habitan el planeta y revisar cómo y qué papel juega la CCS en ellas.

De manera reciente, existe una preocupación por realizar investigación científica que permita analizar la relación de las Tecnologías de Información y Comunicación en las apuestas de cambio social:

En los últimos años, esta área de estudio se ha visto influenciada por un creciente número de trabajos que abordan la influencia de las redes digitales en la transformación social, como por ejemplo en la apropiación que hace la sociedad civil de las diversas herramientas para empoderarse, fomentar sus demandas y facilitar su organización, en el marco de un contexto de movilización social en diferentes países y regiones (Arévalo \& Farné, 2016, pág. 12).

${ }^{5}$ Web Of Science 
Estas reflexiones apuntan a entender los procesos de activismo en el marco de la exigencia de derechos, pero ahora con un componente global que aporta en la preocupación de lo público, entendido el espacio físico como un lugar sin fronteras en el que los problemas locales se discuten en el plano mundial.

En este sentido, a continuación se hace énfasis sobre los nuevos campos de actuación que aparecen en el horizonte inmediato de la comunicación, desde los cuales la CCS tiene retos importantes que enfrentar.

\section{Comunicación y deporte}

Contrario a la tendencia de ubicar la comunicación y el deporte con el periodismo deportivo, este movimiento surgido en los años noventa, ha venido siendo una opción de cambio para jóvenes, que en casos como Colombia, han estado involucrados en procesos de desmovilización, especialmente. Es más que un tema centrado en goles o registro de resultados deportivos. Implica análisis de medios y audiencias, así como co-responsabilidad social de entidades a cargo del deporte local, regional y nacional.

Cárdenas declara a esta actividad social, como un vehículo legítimo para promover la cohesión social, en especial, en sociedades que experimentan pobreza, violencia y conflicto. Múltiples actores como organizaciones no gubernamentales, los países, las instituciones, etc. han tenido en cuenta al deporte como un tema importante de sus agendas. Otra razón para tener en cuenta este campo es la situación de posconflicto en que se encuentra Colombia, “(...) es recomendable que los programas y las intervenciones sociales basados en el deporte sean rediseñados para hacer frente a los retos que esta fase implica" (Cárdenas, 2016, pág., 61).

Angulo viene desarrollando esta iniciativa de la comunicación en la Universidad del Tolima, asegura que la relación comunicación y deporte es estudiada desde la segunda mitad del siglo XX por la historia la sociología y la filosofía, “(...) pero, sin duda, Elias y Dunning (1995) fueron los que caracterizaron la importancia del deporte desde el medioevo hasta nuestros días, tomando como referencias fenómenos como el hooliganismo, la violencia y los procesos civilizatorios" (Angulo, 2016, pág., 107).

Por otra parte, Kassing et al. (2004), resaltan que la comunidad del deporte, además de ser compleja e influyente, constituye un conjunto de interrelaciones y actos de comunicación mediada y no mediada, del que participan entrenadores, atletas, árbitros, medios de comunicación deportivos, clubes, aficionados, patrocinadores organizaciones deportivas, entre otros.

"[En Colombia] todavía no son abundantes las investigaciones sobre el deporte, si las comparamos en cantidad con las que se producen en Brasil, Argentina, Norteamérica, Europa y Oceanía 
(Australia, especialmente)" (Angulo, 2016, pág., 125), lo que para este autor se entiende como un desconocimiento de la importancia que tiene el deporte en "los imaginarios políticos, económicos y sociales del colombiano" (pág., 125).

A estas investigaciones se suma Zarama (2016), quien enmarcada en el postconflicto colombiano reafirma, desde los resultados hallados, las relaciones deporte-cultura-comunidad-jóvenes y alternativas de reconstrucción del tejido social. Además de experiencias como "El Golombiao", que busca promover el afianzamiento de competencias ciudadanas entre los jóvenes y para lo cual se utiliza el fútbol como la forma de convocar, que favorece al establecimiento, cumplimiento y revisión de acuerdos de convivencia (Mena, 2014).

¿Desde dónde se observa o relaciona del deporte y la comunicación para el cambio social? Angulo (2016), entrega algunas pistas al respecto, las cuales se deberán obviamente profundizar y analizar. Una parte desde el ámbito investigativo y otras desde los contextos propios. Del primer campo, Billings (citado por Angulo, 2016) dice:

[Las] cuatro áreas en las que se clasifican los estudios de la comunicación y el deporte y que, en algunos casos, son coincidentes con las de Kassing. Estas son las fundaciones comunitarias y deportivas: estas abordan la forma como los medios de comunicación, sus productores, mensajes y efectos, los fanáticos, las organizaciones deportivas y todas las relaciones interpersonales que desde allí se producen, influyen en el deporte y la sociedad. La identidad en el deporte: se refiere a cómo se construye, mantiene y lucha por la identidad desde escenarios conflictivos como los del género, raza, etnicidad, política, nacionalismo y discapacidad. Las relaciones deportivas: los autores caracterizan las interacciones entre padres e hijos y entrenadores para la socialización deportiva y la consecución del triunfo, así como el manejo de las crisis en las organizaciones deportivas. 4. Las tendencias emergentes en comunicación y deporte: se centran en el estudio e implicaciones de la mercantilización y de los videojuegos o juegos de fantasía en el desarrollo futuro del deporte. (pág., 112).

En consecuencia, la comunicación y el deporte constituyen un nuevo campo en el que fluye o está presente la interdisciplinariedad y en donde la interacción comunicativa es transversal, de ahí que sería factible pensar en orientar sus intereses para el área de la CCS, pues lo indagado muestra que es una línea que examina “...el deporte como una faceta humana que promueva la tolerancia y la convivencia pacífica en tiempos - a veces inciertos- de posconflicto, como ya lo ha propuesto Cárdenas (2016), y que involucre los saberes de la comunicación mediada y no mediada" (Angulo, 2016, pág., 127).

\section{Comunicación e innovación}

Bernardo Alayza Solís de la Universidad Católica del Perú, es en Latinoamérica uno de los representantes de esta línea que pretende aportar a los procesos de innovación, para los cuales el papel 
de la comunicación se ha quedado en transferencia de información vertical, dejando por fuera a los que pueden ser partícipes en prácticas de procesos de innovación.

Al respecto Leeuwis y Aarts (2016), reconocen que eso que nació con la teoría de la difusión de las innovaciones (Rogers, 2013), que cambió de dirección con los aportes de la escuela latinoamericana de comunicación y que ha seguido siendo de interés para los investigadores, deja ver cómo, la comunicación gana un rol más activo en la conformación de redes sociales, micropolíticas y desarrollos científicos orientados a promover cambios sociales a nivel macro.

Los autores establecen además que,

A shift towards sustainability through decentralized energy webs, for example, not only requires new technology and infrastructure, but also new forms of organization among 'prosumers' (i.e. citizens who are no longer only energy consumers, but who also produce energy through e.g. solar panels that are connected to the grid), new contractual arrangements between 'prosumers' and energy companies, new cultural norms, as well as changes in energy legislation and policy. In other words, innovation includes social and institutional innovation (pág. 3).

La investigación acerca de la comunicación para la generación de procesos de innovación inclusiva que lidera Alayza (2017), en Cusco, Perú, es una interesante forma de comprender a la innovación como parte de un proceso social en el que participan diferentes actores que se involucran con esa comunidad y que en su trabajo mancomunado posibilitan la transferencia de conocimiento. El autor considera que ahondar en estos procesos de conexión entre la comunicación y la innovación contribuye a mitigar conflictos relacionados con desarrollo, inequidad y exclusión, que han hecho parte de la historia del desarrollo de los países de Latinoamérica.

En este mismo sentido, Leeuwis y Aarts (2016) establecen que "[the] innovation involves multiple technical and social changes that somehow take place more or less simultaneously in a network of interdependent stakeholders" (pág., 4). Además, afirman que los estudios han demostrado que el éxito de aplicación de una innovación en una unidad poblacional depende en gran medida de dos factores como son: aprender de las dimensiones sociales y culturales de la innovación y la conformación de redes de colaboración tanto para la creación de la innovación como para la formación en el uso de esta.

La comunicación como facilitadora de procesos inclusivos de innovación es entendida desde las interacciones que se establecen entre los actores socialmente situados en un contexto cultural e histórico, en el que se integran, desde un papel activo (coparticipantes) al que aportan en la construcción de cambios sociales que traspasa la mera transferencia de conocimiento (Alayza, 2017). El rol del comunicador aquí se considera desde facilitar entre los diferentes actores, lo que en 
palabras de Leeuwis (2003) es "The emphasis is (...) the facilitation of network building, social learning and conflict management among a variety of actors with a view to arriving at new innovations" (pág., 36).

\section{Comunicación para el cambio social y las TIC}

En palabras de Servaes (2008) el uso de las plataformas digitales crea nuevas formas de organización, de relacionamiento y de interacción para el cambio social, en la medida que la comunicación permite nuevos modos de co-participación, democracia e inclusión, para el desarrollo de la comunidad. Al respecto Harman (2017) consideran "To make possible those processes is necessary to build networks" (pág., 13), redes a través de las cuales se puedan configurar nuevos espacios de diálogo, de trabajo colaborativo, de deliberación, construcción de aprendizajes, en tanto que "su dimensión rizomática e interactiva es hacer referencia a «un espacio complejo de múltiples entradas, recorridos y salidas interrelacionadas y en movimiento»" (Silva citado en Barbas, 2012, pág., 169). Al respecto, algunos de los aspectos en los que se ha venido trabajando son:

\section{- $\quad$ Las multialfabetizacionales de la ciudadanía digital.}

Se ubican en este frente los procesos de alfabetización digital dirigidos a grupos poblaciones tales como indígenas, madres cabeza de familia, reinsertados o desplazados, entre otros. Así lo deja ver la investigación de Restrepo (2011), sobre el programa “Mi llave”, una estrategia para promover procesos de capacitación en competencias laborales y sociales para poblaciones vulnerables de la que participó un grupo de jóvenes colombianos desmovilizados y víctimas de desplazamiento. En el mismo sentido Proenza (2012), en el libro Tecnología y cambio social, presenta casos de estudio sobre el impacto del acceso público a las computadoras e Internet en los países de Argentina, Chile y Perú. El estudio estuvo dirigido a comprender las prácticas cotidianas dentro del proceso de apropiación que hacen los jóvenes de comunidades marginales sobre los espacios públicos de acceso a las tecnologías digitales.

Los nuevos escenarios digitales se integran a las prácticas cotidianas de las comunidades, constituyéndose en soportes desde los que se pueden impulsan procesos de cambio social, a partir de la generación de nuevas prácticas de actuación y de participación. Clemencia Rodríguez (2011) afirma que las TIC son plataformas que dan lugar al fortalecimiento de los medios ciudadanos, entendiendo a estos como espacios de "recodificación del entorno, de recodificación del propio ser, es decir, procesos de constitución de identidades fuertemente arraigadas en lo local, desde dónde proponer visiones de futuros sostenibles, verdes; versiones sí locales, pero no provincianas; es decir, enredadas, conectadas con lo global” (pág., 44). De esta manera, la formación de comunicadores sociales que aborden esta línea debe permitir reconocer en las TIC un nuevo escenario para la información y la deliberación ciudadana de asuntos públicos. 


\section{- La narratuiva transmedia para contar las historias desde la cotidianidad.}

Son varios los trabajos que desde la educación y la comunicación integran a la narrativa transmedia como estrategia para promover cambios sociales. Esta narrativa gana su relevancia en la medida que el lenguaje de lo digital se hace cada vez más de dominio generalizado. De acuerdo con las estadísticas del Global Digital $2019^{6}$ reports, que realiza We are Social de HootSuite, el 75\% de los 430 millones de habitantes que tiene Latinoamérica cuentan con acceso a internet.

García y Simanca (2015), de las universidades de Málaga y Cádiz, respectivamente, ven en la narrativa transmedia una forma de promover cambios locales, en la medida que, desde esta narrativa, las personas hacen parte de un proceso de co-participación, co-producción y co-distribución de las diferentes piezas que componen una historia. Los ciudadanos encuentran en las narrativas y plataformas digitales nuevas formas de contar la realidad alternativa que se vive y a la que las redes de poder (Castells, 2017) no permiten ver, con sus impuesta y planeada realidad del consumo, el individualismo y la excelencia.

El ciudadano actual está llamado a pasar de un papel de consumidor al de people-centered (Jenkins, 2008), es decir, sujetos que más que saber usar el dispositivo desde lo instrumental encuentran en la tecnología digital a su alcance un vehículo para construir, proponer, expresarse, divertirse y transformar su realidad. Así lo que importa es la construcción de vivencias, cambios y alternativas de mejora, más que la promoción del uso de las herramientas tecnologías emergentes. Para García y Simanca (2015) la narrativa transmedia debe ser una estrategia disruptiva, su uso debe estar enfocado al aprovechamiento de las multiplataformas, de las redes sociales que permite, de la forma de trabajo y de conocimiento colectivo que permite internet, de la cultura de la libertad (Castells, 2003).

\section{Comunicación y gobernanza}

Su fundamento teórico está basado en la Comunicación Política, la Comunicación Pública y Gobernanza. Esta triada hace posible que la relaciones ciudadanos y gobernantes supere el ejercicio de rendición de cuentas o publicación de contratación, para iniciar procesos reales de democracia, en busca de transformaciones reales de comunidades locales y regionales.

El planteamiento base es que la comunicación de manera transversal, logre que el gobernante admita y realice su función de exponer a través de diferentes mecanismos los programas o políticas públicas que requiere un municipio o departamento, a fin de superar dificultades enmarcadas en el Plan de Desarrollo. Igualmente implica, la presencia activa del ciudadano, informado y formado en la función pública, dispuesto a dedicarse en algunos momentos a la verificación, discusión y aprobación de los lineamientos que harán posible superar actividades para convertirse en planes

${ }^{6}$ https://wearesocial.com/blog/2019/01/digital-2019-global-internet-use-accelerates 
operativos anuales que tienen fines e indicadores puntuales. En síntesis, hacer viable que la acción de gobernar deje de concebirse como una jerarquía, para reconocer en el otro a un sujeto de derechos y deberes iguales a quién ostenta parcialmente el poder de administrar lo público.

Experiencias investigativas llevadas a cabo en la triada Universidad-Empresa-Estado, tales como el programa Concejo Cómo Vamos (2018), Agenda de Gobierno-Piedecuesta y Periodismo Ciudadano (Wandurraga y Gómez, 2008), presencia activa de Observatorios de Estudios y Análisis de la Opinión Pública en diferentes ciudades del país e indagaciones exploratorias orientadas a conocer las relaciones comunicativas de concejales y líderes comunales (Bohórquez-Pereira y Alguero, 2018) o de los concejales y sus seguidores en redes sociales (Flórez, Celis y Bohórquez-Pereira, 2019) permiten por una parte, mostrar cómo y porqué es necesaria la comunicación de los gobernantes con sus gobernados y cómo estos están pasando del desinterés por la función pública a asumir con mayor responsabilidad su rol como ciudadano. De otro lado, la llegada de las diferentes plataformas virtuales y redes sociales, han permitido más mensajes y diversos modos de expresión (memes, gift, fotos, vídeos), contribuyendo a una conexión profunda y directa entre uno y otro de manera recíproca y constante. En este sentido, el papel de la comunicación cobra relevancia por la posibilidad de fomentar diálogo, espacios virtuales de interacción para lograr que los ciudadanos se empoderen y hagan seguimiento a los compromisos de sus mandatarios y dirigentes políticos.

Será necesaria para esta línea, profundizar y concretar cada uno de los conceptos requeridos en esta triada, donde la comunicación se quiere asumir desde el modelo normativo de Habermas (1987) y en particular lo planteado en la Teoría de Acción Comunicativa, T.A.C.

Garrido (2011) explica acertadamente el pensamiento del representante de la segunda Escuela de Frankfurt:

Para Habermas la acción comunicativa tiene que ver con una determinada concepción de lenguaje y entendimiento: "el concepto de acción comunicativa desarrolla la intuición de que al lenguaje le es inmanente el telos del entendimiento" (1978b, p. 79). Por ello, se propone articularlos como un aspecto central en los aspectos práctico-formales de la teoría de la acción comunicativa con la pretensión de desarrollar una teoría del significado: "En el lenguaje, la dimensión del significado y la dimensión de la validez están internamente unidas la una con la otra” (1978b pág. 80). Es decir, una teoría que permitiera identificar grados de acuerdo según el reconocimiento intersubjetivo de validez de una emisión susceptible de crítica (párr. 9).

Habrá que trabajar en cómo relacionar la realidad local con los componentes que hacen parte de la teoría de Habermas y en particular de la T.A.C. y cómo desde ella pueden encontrarse ciudadano y gobernante para hacer que el significado de lo público, tenga en ellos igual reconocimiento, importancia, trascendencia y respeto. 


\section{Reflexiones finales}

La comunicación como la sociedad es dinámica, cada vez más está involucrada en el accionar de la investigación social, encontrando espacios y escenarios para su ejercicio y cuya presencia supera lo instrumental para aportar a la calidad de vida de los ciudadanos. La proyección está en seguir construyendo el camino que hace más de medio siglo han venido señalando Beltrán, Kaplun, Martín-Barbero, Alfaro, entre muchos otros, un camino lleno de altibajos que reclama la presencia desde el liderazgo de procesos científicos sociales que permitan poner a la comunicación al servicio de las personas para su autoreconocimiento, comprensión de la realidad y generación de cambios verdaderos.

La interrelación de la comunicación con lo digital y la innovación social para promover cambios sociales será válida en la medida que permita la generación de espacios en los cuales los ciudadanos, desde su cotidianidad, actúen en consecuencia para lograr lo que Rodríguez (2011) llama "recodificación del propio ser", sin salir de su contexto, pero integrándose a la dinámica del mundo global. Desde esta perspectiva, el comunicador para el cambio social tendrá la responsabilidad de diseñar estrategias, que más allá de lo instrumental, encuentren en las posibilidades de internet, menos desencuentros y delimitación de grupos, para promover relaciones horizontales que evidencien preocupación por los asuntos públicos, con participación informada y respetuosa para construir consensos alrededor de la diferencia.

La participación de la academia, de forma particular de las facultades de comunicación, en el proceso de formar competencias para el abordaje de la comunicación para el cambio social, debe ser de liderazgo de iniciativas de acción-participación, traspasando la esfera de acompañante, debe buscar desde la formación de los estudiantes una relación con las comunidades que permita generar condiciones de empoderamiento para los colectivos sociales que promuevan sus propios cambios, pasar de ser la voz de los que no tienen voz a entregar a los ciudadanos las herramientas para expresarse, para entrar en la dinámica de co-productores y co-distribuidores de sus mensajes para lograr procesos de transformación social.

La CCS comprende un campo de investigación y prácticas inspirado por referentes latinoamericanos, en ese sentido, es destacable el impulso que la Asociación Latinoamericana de Investigadores de la Comunicación - ALAIC - ha dado a las reflexiones en el campo de la CCS, a través del grupo temático GT12 que se dedica a la discusión en torno a paradigmas y experiencias de comunicación para el desarrollo y el cambio social.

Sin duda, la CCS continuará en una apuesta por indagar sobre las prácticas sociales y la participación como elemento fundamental para generar cambios sociales colectivos. Los desafíos emergentes se centran en las reflexiones que apunten a indagar sobre el reconocimiento de los reclamos 
ciudadanos a nivel global para ser escuchados en el marco de los procesos de cambio social, es decir, en el surgimiento de nuevos actores sociales que se mueven en el escenario de la visibilidad y la diseminación de la información en red para exigir respuestas a la crítica explicita de las políticas públicas, el respeto por los derechos humanos y la inclusión en procesos de gobernabilidad y cambio social.

Lo descrito en este artículo vincula la interdisciplinariedad como condición sine qua non para el fortalecimiento del papel de la comunicación en escenarios de cambio social, de lo contrario seguirá ejerciéndose desde una mirada instrumental que no genera transformación social. El reto para las universidades que forman comunicadores para el cambio social se encuentra en la posibilidad de relacionar el escenario teórico de la comunicación con las prácticas sociales y territoriales para fortalecer la capacidad de reconocimiento y análisis de problemas en comunicación.

\section{REFERENCIAS BIBLIOGRÁFICAS}

Alayza, B. (2017). Communication for Inclusive Innovation: revealing discourses and actions toward more inclusive innovation processes for local development in Perú. Ponencia presentada en el Congreso AIMCR-2017, Cartagena D. C., Colombia.

Alfaro. R. M. (2006). Otra brújula, innovaciones en comunicación y desarrollo. Asociación de Comunicadores, Calandria. Lima, Perú. Recuperado de: https://www.calandria.org.pe/rec_descarga. php?id_rec $=135$

Álvarez, L. (2009). Estilos de conocimiento en los estudios de la comunicación mediática en Colombia. Años 1962 a 1990, Popayán, Colombia, Colección de tesis doctorales Rudecolombia. Fondo Editorial Universidad del Cauca

Angulo, L. (2012). Televisión y periodismo comunitarios en la ruta del Desarrollo Humano: modelos y fundamentos. Ibagué: Universidad del Tolima, Il Gráficas. Recuperado de http://repository. ut.edu.co/bitstream/001/1547/1/RIUT-EBB-spa-2015-Televisi $\%$ C3\%B3n $\% 20 y \% 20$ periodismo\%20 comunitarios $\% 20$ en $\% 201 \mathrm{a} \% 20 \mathrm{ruta} \% 20 \mathrm{del} \% 20$ desarrollo\%20humano.\%20Modelos\%20y\%20 fundamentos.pdf

Angulo, L. (2016). Comunicación y deporte: Un campo integrador para el análisis del fenómeno deportivo. Campos en Ciencias Sociales, 4(1), 101-136. Recuperado de https://revistas.usantotomas. edu.co/index.php/campos/article/view/3376

Area, M; Gros, B \& Marzal, M. (2010). Alfabetizaciones y Tecnologías de la Información y la Comunicación. Madrid: Editorial Síntesis S. A. 
Arévalo, A. \& Farné, A. (2016). Comunicación y cambio social. Un análisis desde la investigación centrado en el periodismo. Cultura, Lenguaje y Representación. 15. 11-19.

Barranquero, A. (2005). Latinoamérica en la ruptura del paradigma de la comunicación para el desarrollo. El recorrido de los pioneros en la búsqueda de alternativas democráticas. Punto Cero, $10(11), 7-22$.

Barranquero, A. Sáez, Ch. (2015). La crítica descolonial y ecológica a la comunicación para el desarrollo y el cambio social. Palabra Clave, 18(1), 41-82. DOI: 10.5294/pacla.2015.18.1.3

Barbas, A. (2012). Educomunicación: Desarrollo, enfoques y desafíos en un mundo interconectado. Foro de Educación, 10(14).

Beltrán. L. R. (2006). La comunicación para el desarrollo en Latinoamérica: Un recuento de medio siglo. Revista Anagramas Rumbos y Sentidos de la Comunicación, 4(8), 53-76.

Bohórquez Pereira, G. (2013). Reflexión sobre el ejercicio del comunicador social y periodista frente a los nuevos retos y escenarios de la profesión. Aportes de la comunicación, 16, 48 - 57.

Bohórquez-Pereira, G. \& Alguero, M. (2018). Concejales y líderes comunitarios de Bucaramanga. Percepciones encontradas en sus relaciones comunicativas. Revista Escribanía, Nueva época, Vol. 16, Número 2. Año 21, 45-57.

Cadavid, A., Gumucio, A. (2014). Pensar desde la experiencia: Comunicación participativa en el cambio social. Bogotá: Corporación Universitaria Minuto de Dios. Facultad de Ciencias de la Comunicación. Bogotá.

Canal, M., Navarro, L. \& Camargo, J. (2015). Comunicación, tejido social y trauma cultural: El caso de la población desplazada de Nueva Venecia en el departamento del Magdalena, Colombia. Verbum, 10(10), 25-47.

Cárdenas, L. (2009). Comunicación y construcción de ciudadanía. Aportes para el Desarrollo. Madrid: Instituto Universitario de Desarrollo y Cooperación, Los Libros de la Catarata, Madrid.

Cárdenas, A. (2016). Fomentando la paz a través del fútbol y otros deportes en contextos de conflicto: El caso de Colombia e Irlanda del Norte. Revista Temas, 3(10), 51 - 62.

Castells, M. (2003). La dimensión cultural de internet. Ponencia impartida en el ciclo de debates 
culturales “Cultura XXI: ¿nueva economía?, ¿nueva sociedad?” organizado por la UOC y el Institut de Cultura del Ayuntamiento de Barcelona. Barcelona: España.

Castells, M. (2017). El panóptico digital. Vanguardia Dossier. 63(1), pp. 74-77.

DIDIS (2011). Lerner: Medios masivos y su papel en el desarrollo. [Entrada de blog]. Recuperado de https://es.wikipedia.org/wiki/Coordinadora_de_Medios_Comunitarios_Populares_y_Educativos_del_ Ecuador

Forero, P.; Hernández, N. \& Ordoñez, J. (2015). Aportes a la construcción de paz desde la comunicación y el tejido social: Caso de los parceleros de Santa Paula (Córdoba). Ciudad de Paz-ando, 8(1). Pp. 194-216. Recuperado de: https://revistas.udistrital.edu.co/index.php/cpaz/article/ view/8552/10598

Flores-Marques, D. (2016). Espacio público, desacuerdos y desigualdades: La expresión pública de los activistas en internet. Observatorio OBS, 10 (No. Especial). Recuperado de http://www. scielo.mec.pt/scielo.php?script=sci_arttext\&pid=S1646-59542016000300004

Flórez, J., Celis, A. \& Bohórquez-Pereira, G. (2019). Twitter como medio de interacción política local. Estudio de caso en Concejo de Bucaramanga. Proyecto de investigación para Dirección de Investigación e Innovación, Universidad Pontificia Bolivariana, sede Bucaramanga.

Fuentes, R. (2010). Investigación de la comunicación: Referentes y condiciones internacionales de un diálogo transversal de saberes. Revista Signo y Pensamiento, 57 (XXIX), 38 - 49.

García, M. \& Simancas, E. (2015). La lucha está en el relato. Movimientos sociales, narrativas transmedia y cambio social. Revista de Estudios Culturales de la Universitat Jaume I, XV, 139 - 151.

Garrido, L. (2011). Habermas y la teoría de la acción comunicativa. Razón y Palabra, 75(1). 1-19.

Gumuncio-Dagron. A. (2001). Haciendo olas: Historias de comunicación participativa para el cambio social. Nex York: Fundación Rockfelleer.

Habermas, J. (1987). Teoría de la acción comunicativa. Volumen 1: Racionalidad de la acción y racionalización social”, Madrid: Taurus, Madrid.

Harman, U. (2017). Communication for community development: the importance of building networks in Latin America. Practice Insights, IACD. 1(7). Disponible en http://www.iacdglobal.org/ wp-content/uploads/2017/10/IACD-PI-7-final.pdf 
Herrera, Á. \& Uruburu, S. (2010). La relación entre comunicación y desarrollo en Colombia. El aporte de la investigación de las facultades de comunicación entre 2000 y 2006. Revista Signo y Pensamiento, 56 (XXIX), 208 - 243.

Herrera., Á. (2011). Investigación en comunicación y desarrollo en Colombia en el siglo XXI: El aporte de las facultades de comunicación. Corporación Universitaria Minuto de Dios; Universidad Santo Tomás; Universidad Nacional Abierta y a Distancia, UNAD. Stanford Libraries. 121-126.

Jenkins, H. (2008). Convergence culture. La cultura de la convergencia de los medios de comunicación. Barcelona: Paidós Ibérica S.A. Barcelona.

Kassing, J.; Billings, A; Brown, R.; Halone, K.; Harrison, K.; Krizek, B.; Mean, L. \& Turman, P. (2004). Communication in the Community of Sport: The Process of Enacting, (Re)Producing, Consuming, and Organizing Sport, Annals of the International Communication Association, 28(1), 373-409, DOI: 10.1080/23808985.2004.11679040

Kaplún, M. (1985). El Comunicador popular. La Habana: Colección INTIYAN Ediciones CIESPAL. Quito, Educador.

Leeuwis, C. (2003). Communication for Rural Innovation. Rethinking Agricultural Extension, Editorial Blackwell Science. Oxford, UK.

Leeuwis, C. y Aarts, N. (2016). Communication as intermediation for socio-technical Innovation. Journal of Science Communication. 15(06), 1-12. DOI: https://doi.org/10.22323/2.15060302

Mejía-Betancur, L. (2014). El arte como herramienta de comunicación para el cambio social: El caso de Medellín. Revista Folio 31, Universidad de Antioquia, 59-74.

Mendivil, C.; Racedo, Y.; Meléndez, K. \& Rosero, J. (2015). El papel de la Comunicación para el cambio social: Empoderamiento y participación en contextos de violencia. Revista Encuentros, Universidad Autónoma del Caribe. 13(1), 11-23. DOI: http://dx.doi.org/10.15665/re.v13i1.345

Montero-Díaz, J.; Cobo, M.; Gutiérrez-Salcedo, M.; Segado-Boj, F. \& Herrera-Viedma, E. (2018). Mapeo científico de la categoría «Comunicación» en WoS (1980-2013). Comunicar. XXVI (55), 81-91. DOI https://doi.org/10.3916/C55-2018-08.

Navarro, L. (2010). Entre esferas públicas y ciudadanía: Las teorías de Arendt, Habermas, Mouffe aplicadas a la comunicación para el cambio social. Barranquilla: Ediciones Uninorte. Barranquilla, Colombia. 
Navarro-Díaz, L. \& Aguilar, D. (2015). Las historias de Palenque empiezan en la calle: Jóvenes, comunicación y cambio social. Revista Nómadas, Universidad Central, 43 (1), 250-265.

Navarro-Díaz, L. (2017). Palenque. Comunicación, territorio y resistencia. Ediciones Uninorte. Barranquilla, Colombia.

Pereira J. (2005). La comunicación: Como un campo de conocimiento en construcción - reflexiones sobre la Comunicación Social en Colombia. Revista Investigación y Desarrollo. 13 (2), 412 - 441.

Proenza, F. (Ed.). (2012). Tecnología y cambio social: El impacto del acceso público a las computadoras e internet en Argentina, Chile y Perú. Lima: IDRC-CRDI, IEP. La Plata: Instituto de Investigaciones en Humanidades y Ciencias Sociales (UNLP-CONICET), Universidad de la Plata. Recuperado de https://idl-bnc-idrc.dspacedirect.org/handle/10625/51521

Restrepo, N. (2011). Comunicación para el cambio social y nuevas tecnologías de la información y la comunicación. Etic@net,9(11),158-178.

Rodríguez, C. (2011). Trayectoria de un recorrido: Comunicación y cambio social en América Latina. En Pereira, J. M. y Cadavid, A. (Ed.), Comunicación, desarrollo y cambio social. (37 - 56). Bogotá: Pontificia Universidad Javeriana: Universidad Minuto de Dios: UNESCO.

Roveda, A. (2006). Las facultades de comunicación y periodismo de Colombia: Entre las incertidumbres de la cientificidad y la claridad de las prácticas. Ponencia en el XXIII Encuentro Académico de la Asociación Colombiana de Facultades y Programas universitarios en Comunicación e Información-AFACOM, Caldas, Antioquia. Recuperado de http://www.redalyc.org/ pdf/695/69520212.pdf

Rueda, O. (2006) Diagnóstico de comunicación participativa en el Magdalena Medio. Corporación de Desarrollo y Paz del Magdalena Medio - Universidad Pontificia Bolivariana de Bucaramanga, Bucaramanga - Santander.

Servaes, J. (2008). Communication for Development and Social Change. Francia: UNESCO. Recuperado de: http://citeseerx.ist.psu.edu/viewdoc/download?doi=10.1.1.454.3418\&rep=rep1\&type $=$ pdf

Sforzin, V. (2016). Redes sociales en Latinoamérica. De los usos a las estrategias colectivas. IX Jornadas de Sociología de la UNLP, 5 al 7 de diciembre de 2016, Ensenada, Argentina. En Memoria Académica. Recuperado de: http://www.memoria.fahce.unlp.edu.ar/trab_eventos/ev.8858/ ev.8858.pdf 
Villasante, T. (2015). Metodologías ¿Para qué?, ¿Para quién? En la dinámica del contacto. Movilidad, encuentro y conflicto en las relaciones interculturales. II Training Seminario de jóvenes investigadores en Dinámicas Interulturales. Panel V. Metodologías de investigación intercultural: Experiencias, dificultades, estrategias. Fundación CIDOB. Barcelona, España. Pág. 215 - 225. Recuperado de: https://www.cidob.org/es/publicaciones/serie_de_publicacion/monografias/monografias/la_ dinamica_del_contacto_movilidad_encuentro_y_conflicto_en_las_relaciones_interculturales

Wandurraga, S. \& Gómez, C. (2008). Construcción de agenda ciudadana en el municipio de Piedecuesta para las elecciones de alcaldía periodo 2008 - 2011(Tesis de pregrado). Universidad Pontificia Bolivarana, Floridablanca, Santander. Recuperado de https://biblioteca.bucaramanga. upb.edu.co/docs/digital_16426.pdf

Zarama, P. (2016). El deporte como proceso de comunicación para la creación de escenarios de convivencia en el trabajo con jóvenes en el municipio de Galapa, Atlántico. Trabajo de grado. Pontificia Universidad Javeriana, Bogotá: Colombia. 